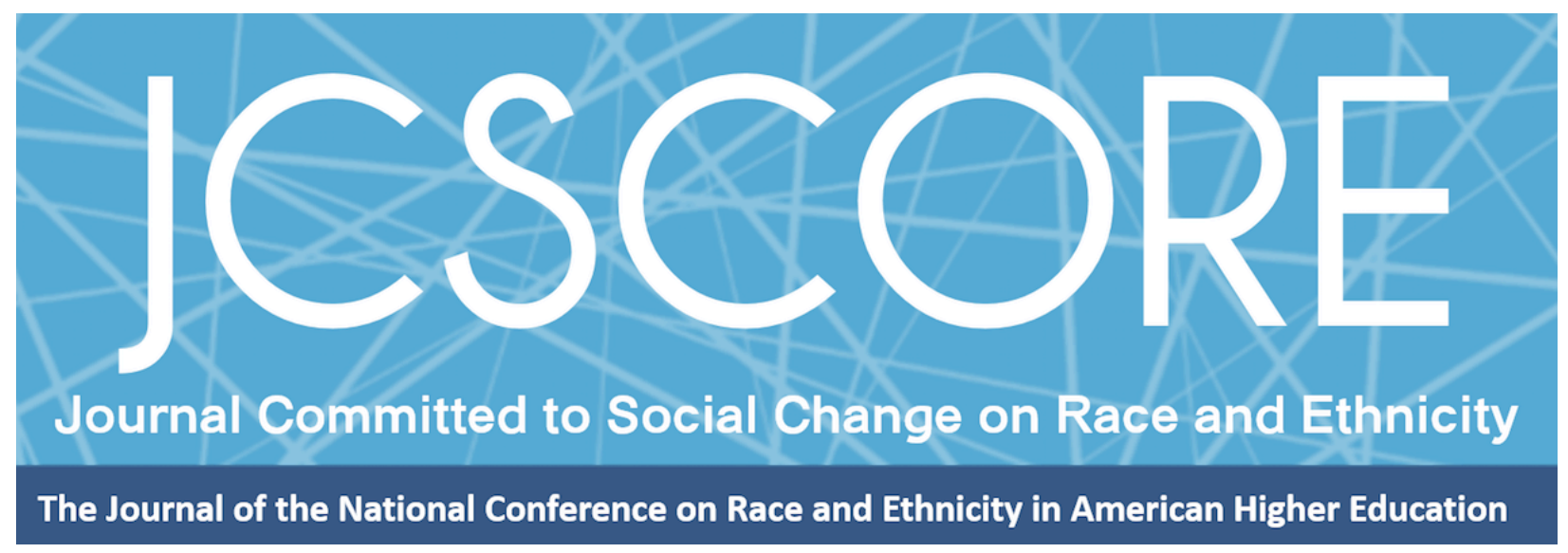

\title{
ON A CONTINUUM: EXAMINING NCORE'S INFLUENCE IN THE DEVELOPMENT OF MULTICULTURAL COMPETENCE IN STUDENT AFFAIRS EDUCATORS
}

Jennifer L. Plagman-Galvin

lowa State University

Ann M. Gansemer-Topf

lowa State University

Journal Committed to Social Change on Race and Ethnicity

Volume 4, Issue 1 | 2018

Copyright ( 2018 Board of Regents of The University of Oklahoma on behalf of the Southwest Center for Human Relations Studies.

Permission of the Publisher is required for resale or distribution and for all derivative works, including compilations and translations. Quoting small sections of text is allowed as long as there is appropriate attribution. 


\title{
On a Continuum: Examining NCORE's Influence in the Development of Multicultural Competence in Student Affairs Educators
}

\author{
Jennifer L. Plagman-Galvin \\ lowa State University \\ Ann M. Gansemer-Topf \\ lowa State University
}

Multiculturally competent student affairs educators are required to effectively meet the needs of today's diverse student population. However, little is known about how educators acquire these skills. This study examined the multicultural awareness, knowledge, and skills gained by student affairs educators through their participation in NCORE, an experiential conference exploring race and ethnicity. Results of the study have implications for graduate preparation programs and professional development opportunities for student affairs educators.

As the racial diversity of college students increases, student affairs educators need to be multiculturally competent (Harper, 2008; Hurtado \& Guillermo-Wann, 2013). Racial tensions, debates and controversies on college campuses have been heightened as a result of college administrations' lack of awareness, knowledge and skills; reiterating the need for multiculturally competent student affairs educators (Pope, Reynolds \& Mueller, 2014).

Multicultural competency is a stated value of the student affairs profession and aligns with higher education goals (Bresciani, 2008). Student affairs educators must acquire the necessary awareness, knowledge and skills to best serve their students (Hurtado \& Guillermo-Wann, 2013; Pope \& Reynolds, 1997); and they benefit from competency development regardless of serving in entry-level, mid-level, or senior-level positions (Howard-Hamilton, Richardson \& Shuford, 1998; Pope, Reynolds \& Mueller, 
Journal Committed to Social Change on Race and Ethnicity | 2018

2004). Because achieving and sustaining multicultural competence requires ongoing professional development, institutions and professional organizations provide opportunities for student affairs educators to develop their competence. The National Conference on Race and Ethnicity (NCORE) is one of these opportunities.

NCORE is "a forum for building skills, alliances, and knowledge about issues of race and ethnicity in higher education," by offering approximately 350 sessions on race and ethnicity in higher education (NCORE, 2018, para. 1). Conference participants comprise senior-level administrators, student affairs educators, faculty and students from more than 1,000 institutions and organizations (NCORE, 2018). The weeklong conference includes keynote speakers, workshops and special sessions related to issues of race and ethnicity. Student affairs educators from this study who participated in NCORE met several times prior to attending the conference, engaged in intergroup dialogue with a cohort of their peers during the conference, and continued to meet once they returned to campus. As part of the cohort experience, they were exposed to readings and discussions, supported undergraduate students attending the conference, and engaged with other experts, educators and students from across the country.

Attending the conference requires a significant investment of individuals' and institutional time and resources, but little empirical research has been done to investigate the influence of this conference on student affairs educators' multicultural competence. One research question guided our study: What multicultural, awareness, knowledge and skills do student affairs educators develop by participating in NCORE, an experiential conference exploring race and ethnicity? Information gained through this study can document the influence of NCORE on multicultural competence 
Journal Committed to Social Change on Race and Ethnicity | 2018

specifically, but also provide broader insights into the development of multicultural competencies for student affairs educators.

\section{Conceptual Framework}

Sue's (2001) Multidimensional Model of Cultural Competence (MDCC) was used as the framework for this study. We used the definition of multicultural competence introduced by Reynolds and Pope (1997) and further definitions of multicultural awareness, multicultural knowledge and multicultural skills introduced by King and Howard-Hamilton (2003). The MDCC outlines three dimensions important in addressing cultural competency in the counseling field: (a) the diversity of human differences including but not limited to race, gender, disability, age and sexual orientation, (b) components of cultural competence including awareness of attitudes, knowledge and skills, and (c) foci of cultural competence at the individual, professional, organizational and societal levels. The student affairs educators who were chosen to participate in NCORE had demonstrated competencies at the first dimension as they had an awareness of student differences. Therefore, we were interested in how NCORE influenced awareness, knowledge and skills; and how these were manifested in the individual, professional, organizational and society levels. This study focused specifically on the second dimension including awareness of attitudes, knowledge and skills.

For the purpose of this study, the definition of multicultural competence for student affairs educators introduced by Pope and Reynolds (1997) was used, defined as "the awareness, knowledge, and skills necessary to work effectively and ethically across cultural differences" (p. 270). The researchers used definitions for multicultural 
awareness, multicultural knowledge and multicultural skills further refined by King and Howard-Hamilton (2003) to investigate how NCORE influenced the multicultural competence of student affairs professionals.

Multicultural Awareness: "Awareness of how people's attitudes, beliefs, values, assumptions, and self-awareness affect the ways they interact with those who are culturally different from themselves." (King \& Howard-Hamilton, 2003, p. 124)

Multicultural Knowledge: "Having an informed understanding of cultures that are different from one's own culture, including knowledge of their histories, traditions, values, practices, and so forth." (King \& Howard-Hamilton, 2003, p. 123)

Multicultural Skills: "Skills that individuals use to engage in effective and meaningful interactions with those who are from different cultural backgrounds than their own." (King \& Howard-Hamilton, 2003, p. 123)

\section{Awareness}

Culturally competent student affairs professionals must be cognizant that students represent diverse worldviews, lived experiences and identity groups; and in many cases, these views will be different from the student affairs professional's worldview, lived experience, or own identity groups (Harper, 2008; Pope \& Reynolds, 1997). Student affairs professionals must be aware of their own attitudes, beliefs, emotions and prejudices and how these influence their work with a diverse student body. Multiculturally aware student affairs professionals are needed to contribute to the overarching goals of higher education, which include inclusive campus environments where students persist and are prepared to navigate a global society.

\section{Knowledge}

The student affairs professional must understand societal structures and policies that are rooted in Colonial-America history, culture and values; and have an understanding of how these structures and policies might work in conflict with the 
Journal Committed to Social Change on Race and Ethnicity | 2018

students they serve (Davis \& Harris, 2013). Knowledge of the history of higher education, federal, state, institutional policies and programs, and the influence of these for diverse student populations is critical to meet the needs of a diverse student body (Harper 2008; Hurtado \& Guillermo-Wann, 2013).

\section{Skills}

Student affairs educators must also have multicultural skills to develop and manage differences at varied levels including individuals, institutions, communities and society (Watt \& Linley, 2013). Watt and Linley define multicultural initiatives as taking actions that disrupt structural inequities limiting the experiences of marginalized groups, while privileging others. They approach multicultural initiatives, and needed multicultural skills of student affairs educators, from a framework that diversity is a social value and outlines three principles that should guide multicultural work including:

(a) a multilevel transformational approach, (b) engaging the head, heart and hands, and (c) alignment with stated goals and outcomes.

\section{Methods}

In order to explore multicultural competencies of student affairs educators, we conducted a qualitative case study at a large, predominately White research-intensive public institution located in the Midwest. The case study design investigated multicultural awareness, multicultural knowledge and multicultural skills of student affairs educators attending NCORE, an experiential conference exploring race and ethnicity. A case study design involves the collection of detailed information within time and activity parameters (Stake, 1995; Yin, 2009). This case study was bound by parameters of both time and activity. The time parameter included the beginning and 
conclusion of the conference experience of the student affairs educators as they prepared for, attended and reflected on their NCORE experience. The activity was the NCORE conference and the immersion experiences leading up to, during, and following the conference.

Yin (2009) outlines three conditions for a case study: (a) research questions are in the form of how or why, (b) the phenomena is independent of the researcher's control, and (c) the phenomena is current rather than historical in nature. This case study meets all three conditions outlined by Yin. The research questions that inform this study are in the form of how. The phenomena, multicultural competency development, is independent of the researcher's control and a separate process from this study. The phenomena of multicultural competency development is relevant for institutions of higher education, as well as greater society.

Qualitative case studies are particularistic, descriptive and heuristic in nature (Merriam, 1997). The particularistic element of a case studies focus on a situation, event, program or phenomenon (Merriam, 1997); and in this case the phenomenon is the development of multicultural competence. Case studies are descriptive as rich, thick description of the phenomenon is collected. This study collected rich, thick description about the phenomena of multicultural competency development. Finally, case studies are heuristic, or bring meaning to the phenomena; wherein this case study investigated the meaning of multicultural competency development as interpreted by the student affairs educators participating in this study. 
Journal Committed to Social Change on Race and Ethnicity | 2018

\section{Participants}

Student affairs professionals participating in NCORE received an invitation to participate in the study. Of the 23 possible participants, 17 chose to participate. Participants were grouped by the number of years they had attended NCORE: seven individuals were attending their first NCORE (novice), six were attending for the second or third time (returners), and four had attended four or more times (experts). Eight identified as ALANA and nine identified as White; 13 identified as female and four identified as male. Participants represented a range of tenure in student affairs from new professionals (five), mid-level professionals (six) to seasoned professionals (six). Participants represented 12 distinct units across both academic and student affairs. This variety in backgrounds, experiences, and job responsibilities allowed us to identify commonalities and differences in the influences of the NCORE experience on multicultural competence development.

\section{Data Collection}

Data were collected through semi-structured interviews and focus groups (Jones, Torres \& Arminio, 2014; Maxwell, 2013), field notes (Rossman \& Rallis, 2012) and interpretive memos (Seidman, 2013). Participants were invited to a set of pre- and post- interviews or focus groups relative to their assigned category for years participating in NCORE.

As this study suggests, multicultural competence development occurs along a continuum. Focus groups with the novice and expert groups were appropriate in that first-time attendees were able to discuss their personal and professional goals for attending, apprehensions about exploring issues of race and ethnicity, and they were 
able to reflect together on their first NCORE experience. Expert participants, those attending NCORE four or more years, were able to reflect on their own multicultural competency development over several years, building from one another's diverse vantage points (Jones et al., 2014; Rossman \& Rallis, 2012). The semi-structured oneon-one interviews with the returners provided opportunity for deeper exploration into their multicultural competency development and flexibility for clarification and follow-up questions. The returners were not new to the experience, but were also not seasoned NCORE participants either, as they were processing and making sense of the meaning of their own development.

\section{Data Analysis}

After data collocation, we analyzed the data following Saldaña's (2015) methods for coding qualitative research including collecting data, coding data, identifying patterns, and categorizing the patterns. We began data analysis by writing analytic memos to capture insights and reflection immediately following each interview, including emerging themes, methodological questions, connections between themes, literature, and theory (Rossman \& Rallis, 2012). Simultaneous to completing interpretive memos, we transcribed audio-recordings verbatim after each interview. The cleaned and deidentified transcripts were sent to each research participant for validation.

We analyzed the cleaned and de-identified transcripts and analytic memos utilizing a three-phase approach (Saldaña, 2015). In the first phase, we looked for themes around multicultural awareness, multicultural knowledge and multicultural skills. In the second phase, we looked at the data within each of the three competency areas for themes within the overarching themes of multicultural awareness, multicultural 
knowledge and multicultural skills. Finally, in the third phase, we looked at emerging themes that supported how student affairs educators develop multicultural competencies. Four higher education peers were engaged in the review of the data for validation of the themes, bias, discrepant evidence, and any other ethical issues. Peer feedback was incorporated into the final analysis.

\section{Researcher Positionality}

Creswell (2014) encourages qualitative researchers to clarify their own biases, and how those biases might influence the methodology of the study. We acknowledge our positionality as researchers. The investigator for this study identified as a White female with more than 20 years of experience in higher education and the nonprofit sector as a student affairs educator and academic administrator. The co-author identified as a White, first-generation female, with 20 years of experience working in higher education and student transitions prior to becoming a faculty member. As a student affairs administrator and faculty member, we work to acknowledge our privilege as White women, believe in the importance of developing our multicultural competence, and seek opportunities to develop in these areas. We were mindful of potential bias and practiced reflexivity to ensure previous experiences did not impede the research process (Creswell, 2014).

Neither of us had attended NCORE prior to this study. We knew several colleagues, both faculty and student affairs educators, that had attended NCORE. From their feedback, we understood that the NCORE experience was important to their work in higher education. We were interested in learning more about the 
Journal Committed to Social Change on Race and Ethnicity | 2018

experiences of our colleagues, thus the impetus for this research. One of the authors has attended NCORE.

\section{Limitations}

This study was not without limitations. It was conducted at a predominately White institution (PWI) located in the Midwest. Participants contextualized their multicultural competency development as professionals within the environment of a PWI. Participation in the study was limited to only one cohort at one institution. Additionally, not all student affairs professional invited to the study chose to participate; the experiences of cohort members that did not participate may have yielded different findings. We also only focused on those attending NCORE and did not examine experiences of other student affairs educators who had participated in other programs with similar goals. We limited the scope of the study to the second dimension of the MDCC framework; choosing to focus on cultural competencies without the intersection of multiple worldviews (first dimension) or areas of foci (third dimension).

\section{Results}

Our study sought to understand the multicultural awareness, knowledge, and skills student affairs educators develop by participating in an experiential program focused on race and ethnicity. Findings indicated that through participation in NCORE, student affairs educators do develop multicultural competencies in the form of awareness, knowledge and skills. They suggested their multicultural competencies developed along a continuum beginning with multicultural awareness, followed by knowledge and eventually skills. Participants suggested that multicultural competency 
Journal Committed to Social Change on Race and Ethnicity | 2018

development is a lifelong journey that includes self-development, professional development and the ability to influence others' multicultural competencies.

\section{Multicultural Awareness}

All of the participants from this study suggested that the NCORE experience influenced their multicultural awareness including their attitudes, beliefs/assumptions, self-awareness and awareness of one's own privilege.

Attitudes. NCORE broadened Elena, Thomas and Anna's attitude toward race and ethnicity. Anna, a veteran participant, shared that NCORE has expanded her worldview about issues of race and ethnicity. "I feel like [NCORE] has really broadened me in a lot of ways, I think even how I see the world." For Thomas, a second-year participant, NCORE developed his awareness through reflection and making meaning

of his experiences. "NCORE causes me to pause more, reflect more, to be much more reflective about things that I see; that I hear." Elena, a first-year participant, shared her attitude toward learning about race and ethnicity, "The minute you think you are aware or know all of these things ... is when you should retire and go home. . I can't imagine knowing anybody's lived experience other than my own."

Beliefs and assumptions. Participants shared that NCORE influenced their beliefs and assumptions in ways that affect their interaction with others culturally different from themselves. In particular, beliefs and assumptions about students they serve and their campus environment. Joe shared that NCORE influenced his awareness and understanding of underrepresented students, "I'm more in tune with our student struggles." Sarah corroborated Joe's perspective, "In terms of awareness, [I experienced] a lot of ah-ha moments that really made sense with what I was learning 
about the issues that African American students probably feel on this campus." Nou shared her belief about the political nature of working through issues of race and ethnicity in higher education.

I am an unafraid educator for our undocumented students, but forgetting it is also a political statement you are making. It's not just l'm going to support these students. You are politically saying I support these issues that are unconstitutional.

Nou became aware of her beliefs and assumptions about the political nature of advocating for undocumented students through their journey in higher education.

Self-awareness. Participants shared that once they attended NCORE their selfawareness was permanently influenced; especially as they engaged in self-reflection and interacted with others different from themselves, as shared by Kim.

Paying attention to how I am received. The skin that I'm in. My tone. My communication style. Making sure l'm being accommodating to the person I'm interacting with, because this may be the first time that they've interacted with someone like me.

Privilege was unilaterally identified by participants when asked how NCORE influenced their awareness of others culturally different from themselves. Participants shared they became more self-aware of their own privileges through the NCORE experience. Joe shared how NCORE influenced self-awareness of his privilege.

I probably [have more privileges than the majority of NCORE participants] ... The reality for me to be able to say I didn't earn any of it is what NCORE, and this process, and this awareness has let me do.

Participants recognized their own privileges, regardless of identifying as White or underrepresented. For example, Nou attended a session about undocumented immigrants at her first NCORE, she reflected on her understanding of the privileges she has despite not being a member of many historically privileged groups. 
[NCORE] really struck a chord with me. It made me think about my own privileges, my own privilege as a Woman of Color. Being born in this country. Having U.S. citizenship. My parents going through and getting their citizenships and just thinking wow - this is such a privilege to sit here and say that I'm an American citizen, when someone who maybe has grown up in this country doesn't have that privilege.

Ben was also reflective of his privilege, "As a White person, my whole life has been built around me in America. Everything was made for me - the education system, healthcare, college, scholarships."

The NCORE experience provided for Joe, Nou and Ben an awareness of their own privileges, regardless of if they identified with multiple privilege groups, or only a few. NCORE provided them the opportunity to explore their own racial and ethnic identities.

\section{Multicultural Knowledge}

Participants suggested that they began to learn about the history, traditions, values and practices of cultures other than their own within the context of higher education through their NCORE experience.

History. Participants emphasized their expanded knowledge about U.S. history for people of traditionally underrepresented races and ethnicities. Phryne shared an example of learning history written from the lens of a White historian and likely shared by White educators for White audiences.

In graduate school I was taught about how amazing the G.I. Bill was for providing access to higher education. But over a million Black soldiers that served in WWII were not given the opportunity of receiving the GI Bill. So how is that access?

Phryne's example of learning about history was similar to other participants who shared examples of how their previous history was disseminated through a lens of privilege. 
Journal Committed to Social Change on Race and Ethnicity | 2018

NCORE provided alternative perspectives on historical events and their impact on underrepresented groups in America.

Traditions and Values. Participants affirmed they gained knowledge about cultural identities including African American, Asian American, Latinx, Indigenous and White. Olivia, Sarah and Thomas each shared examples of attending sessions where they learned more about specific racial and ethnic identities. Olivia attended an African American session on post-traumatic slave syndrome that inspired her to buy a book and have further dialogue, "Let's talk about the historical implications of what this means." Sarah provided an example of learning about the Asian American culture, "I didn't know a lot of the terms they were talking about. Even Asian American identity models were brand new to me." Thomas gained knowledge about Indigenous cultures and realized he had much more to learn, "I have a much better understanding of the term Native American [Indigenous] is not reflective of that group. I mean that group is tribes, and they are all different." For some of the participants, NCORE confirmed what they thought they knew about race and ethnicity, for others NCORE expanded the foundation of knowledge that they already possessed, and yet for others NCORE contradicted assumptions that they previously held about race and ethnicity.

Practices. The topic of undocumented students in higher education was a salient focal point for participants, in particular higher education practices related to undocumented students. Nou shared that her knowledge about undocumented students was clarified and expanded, "People would ask me before NCORE about 
undocumented students. I had an idea, but I didn't really know. I know so much more than I did before I went to NCORE." Elena supported Nou's example, "I had no idea. [Before NCORE] I would have talked about Dreamers." Olivia shared that her perspective prior to NCORE was about Latinx undocumented students, but through NCORE became knowledgeable about immigration stories from cultures other than Latinx.

A lot of time when we talk about undocumented people [in America], we think of those coming from South America. . There are all of these different stories that are happening. When we talk about 'the border', what about the rest of the borders?

Thomas, along with other participants, acknowledged limited institutional infrastructure to support undocumented students.

I went to a couple of sessions that dealt with undocumented students, because I know that we have a significant population of undocumented students. I'm not thoroughly convinced that we have the support structures in place and that we know how to work with those students.

For Nou, Elena, Olivia and Thomas their knowledge about undocumented students was broadened, and some participants such as Thomas had begun to consider implications for higher education practices.

Developing multicultural knowledge. Participant shared the conference influenced their multicultural knowledge, but the knowledge they gained was only a foundation for areas of further exploration as shared by Heather, "I have so many notes on things that I need to research." Olivia described a similar perspective.

[NCORE] is still only five days. It increases your knowledge. It opens the door, but you have to be willing to step in. It is going to start exposing you to things, but you really have to still delve deeper. 
As participants attended sessions at NCORE, they became cognizant there was likely much more multicultural knowledge they had yet to acquire. They suggested the NCORE experience was a source of multicultural knowledge that opened doors for further exploration of issues of race and ethnicity, and they were responsible for continuing to develop their multicultural knowledge post NCORE.

\section{Multicultural Skills}

Participants who shared skill development were primarily returners and veterans to the NCORE conference. They identified recognizing diverse perspectives, communicating, and influencing others as three skills they developed by participating in NCORE and engaged in multicultural work over time.

Recognizing diverse perspectives. Understanding multiple perspectives from diverse life experiences, was a theme carried through from areas of multicultural awareness and knowledge development, to skill development. As Joe described, "This program has afforded me the opportunity to see the issue through so many different lenses." Sarah shared, "Every year it has an impact on you. You come back with a little bit of a different lens ... You're seeing things differently." Participants provided examples of using awareness and knowledge gained about different racial and ethnic identities in their skill-set to engage with others, as shared by Anna.

All of the stereotypes [theories or ethnic characteristics] you would assume about people. You have to pull that back, and just wait for the story to unfold. Because, yes, they may have these things in common, but this is where it is different.

Anna, and others, recognized students have unique lived experiences and student affairs educators need to understand multiple lenses when working with students rather than making assumptions about student's experiences and backgrounds. 
Communication. Participants suggested their multicultural communications skills were influenced through NCORE as they developed the competencies to listen, dialogue with others, facilitate dialogue, and use relevant language.

Listen. Kim shared she learned the importance of listening through her NCORE experience, "I do listen. I picked that skill up very intently. . . You're in the zone, you're already charged. But the best thing you can do is listen." Kim had developed the skills to identify when to listen to others' experiences and perspectives, and when to engage in the dialogue about race and racism.

Danielle described how she began to listen to the life experiences of others through her NCORE experience, "I went to the [NCORE] sessions with the lens of storytelling and listening for people's stories." Thomas suggested he better listens to students because of his NCORE experience.

I can listen with a more informed ear. . I I understand what they are saying, because this sounds like some of the information that I learned at the session I went to on Asian Americans. . I can help students by saying, "You know, I'm not living your experience, but I understand it."

Kim, Danielle and Thomas learned to listen to the stories of others; but also to understand and comprehend the meaning of those experiences.

Dialogue. Claire, who had attended several NCOREs, shared how these experiences helped to develop her skill-set when dialoguing with students and their families in her job.

I've had a lot of those conversations with families over time [regarding issues of race and ethnicity]. I think my exposure, and self-knowledge and teaching and learning. ... the whole NCORE process has made me much more open and willing to have those conversations.

Participants emphasized their willingness to have meaningful conversations about race and ethnicity with others because of their NCORE experience. 
Facilitate. Participants also reported the ability to provide leadership in the form of facilitating dialogue related to issues of race and ethnicity. Sarah shared that by modeling colleagues she viewed as expert facilitators, she developed skills to facilitate difficult dialogue. "Every year, because of practice, [my facilitation] gets a little bit better." Olivia shared examples of facilitating dialogue about race and ethnicity in her department, "Because of NCORE I'm trying to facilitate more conversations around issues of race and ethnicity [with colleagues], and a lot of other issues of inclusion." Joe shared an example of facilitating dialogue as a supervisor with his staff team, "I've made the decision to introduce and require my staff to read [a book about race and ethnicity]. It's a professional development exercise that I'm going to continue the dialogue."

Sarah, Olivia and Joe developed the skills to influence the multicultural competence of others as a result of attending multiple NCOREs.

Language. NCORE provided participants with the most current language used within the discipline of race and ethnicity. Renee shared she expanded her vocabulary, "[l learned] a lot of new terms and a lot of old terms that are used differently." LaYin noted the importance of correct language when working with students about race and ethnicity, "When we're working with a network of students, what language do we bring?" Olivia illustrated the importance of having the correct language when making observations about issues related to race and ethnicity in her work environment. "To have the language ... and be able to put your finger on it." NCORE provided for Olivia the language, terminology and definitions to describe observations of racism and cultural conflict within her professional setting.

Influencing others. The multicultural skills of veteran participants included developing their own multicultural competencies, as well as the skills to influence 
multicultural competencies of students and colleagues. Kim shared an example of developing multicultural competencies with her staff, "A great part of attending the conference was being able to bring back an instrument ... to do some personal reflection [with staff] about [communication styles and conflict]." Claire shared an example of being aware of policy changes and influencing others within her sphere of colleagues regarding the potential impact of policy changes for underrepresented populations at her institution.

Is anybody [in administration] considering how [a new policy] is going to impact Students of Color? How this is going to impact low-income students? How is it going to impact women? ... I have the ability to raise the questions and at least make somebody hear.

LaYin provided an example of her work with a student organization that is an awareness coalition, "Learning about history of activists and groups from different races and ethnicities, understanding that all oppression is connected, finding articles to share with them, and having discussions to help them reflect and think." Sarah provided an example of influencing students she teaches by integrating influences of race and ethnicity into curriculum, "How I teach students, or interact with them. I think for me [NCORE] opens doors for resources for teaching them."

\section{Continuum of Multicultural Competency Development}

We began this study focusing on awareness, knowledge, and skills of multicultural competence. Although we were interested in examining each as distinct aspects of competence, participants frequently discussed this development along a continuum. They first developed multicultural awareness, followed by multicultural knowledge. Multicultural awareness and knowledge evolved into multicultural skills. Thomas provided his perspective of having first gained awareness. 
I look at awareness as almost a first step to knowledge. I'm more aware of the issues of [Indigenous People] and their culture, but I don't consider myself knowledgeable because I haven't taken that next step of deeper exploration.

As Thomas described, he became aware of the Indigenous culture and his awareness developed into knowledge; yet he did not consider himself knowledgeable by solely participating in the NCORE experience. He suggested he needed to seek additional information and engage with Indigenous people before he felt truly knowledgeable about the culture.

First time participants questioned their multicultural knowledge prior to NCORE, but following NCORE could more clearly articulate examples of knowledge gained and how the additional knowledge might transition to deeper understanding and skill development around issues of race and ethnicity. Prior to the conference Danielle illustrated her lack of confidence in her own multicultural competencies, "If I hear my peers talking about different theories ... I feel almost intimidated. I don't know all these theories." Following the conference, Danielle shared, "I learned more about some theories. I'm sure some of those theories could change how I work with students." Following NCORE Danielle reflected on knowledge gained from the experience and suggested what she learned would influence her work with students.

Student affairs educators who had attended more than one NCORE suggested their multicultural knowledge expanded and became deeper each year they participated, as shared by LaYin.

I think after attending [multiple] NCOREs and the sessions, I now have different perspectives in my memory bank. I'm able to utilize what l've learned from different theorists, and different presenters, and different literature. It is not just 'what I know', but here is what I know based on multiple things. 
LaYin began to transition from having basic knowledge, to understanding the premise and supporting data for the knowledge she had acquired.

Sarah shared in graduate school she developed multicultural awareness, but did not yet have the knowledge to support her awareness, "I remember in grad school learning all of these things, but being really frustrated because I could never get my point across. I think in terms of knowledge it gives me the meat to back-up the claims." Sarah suggested she developed multicultural awareness in graduate school, but several years after graduate school it was her participation in several NCOREs that influenced her multicultural knowledge.

Much like knowledge, the data indicated skill development followed a continuum of increased cultural competence associated with more time involved with NCORE. First-year NCORE participants were less likely to identify or articulate skill development as a multicultural competency influenced by NCORE as described by Ben, a first-time conference participant.

Skills is hard to say. Maybe at some point l'll kind of tease out some skills. .. I definitely got some information on how do you talk about whiteness with White people. I wouldn't say that's a skill, because that's not a skill I have at this point, but I have the information.

Ben's description of how NCORE influenced his multicultural skills was comparable to other first-time NCORE participants.

Even though there were sessions specifically addressing multicultural awareness, knowledge, and skills; when asked about the influence of NCORE on their multicultural competencies, first-time participants were more likely to provide examples of increased multicultural awareness. They expressed their multicultural knowledge 
was influenced but needed to be further expanded before they would describe themselves as knowledgeable. They were least likely to identify, or clearly articulate, examples of how NCORE influenced their skills. Elena's perspective supported Ben's observation, "Skills, I don't have any answers. I think that's what the continual process is supposed to be as we come back and we continue to meet." Elena indicated her first NCORE experience did not necessarily equip her with multicultural skills; rather she indicated returning to campus, continuing to meet with her colleagues and applying her acquired awareness and knowledge to her work might evolve into multicultural skills.

Veteran participants shared that multicultural competency is a lifelong journey. Joe, although he had been involved in NCORE for several years, still acknowledged opportunity for multicultural competency development.

I've literally had to almost flip the script. . I still have racist tendencies. I still have prejudice tendencies. . . I don't take that away from my history, and I don't apologize for it; but I recognize it.

Like Joe, other veteran participants discussed their journey in developing multicultural competencies. Anna related her journey to work with students, "Where are we at in our own walk or journey? And how does that affect how we work with students?" Anna recognized that she was continuously developing as a person and a professional, and recognized her own development influenced her work with students. Olivia also discussed her journey as it related to students and their development, "Working with [underrepresented] students ... through this journey and finding their place. Feeling like they have a sense of belonging." 
Claire, another veteran participant, recognized that as her multicultural competencies evolved after several years participating in NCORE, she became the colleague counted on to work with underrepresented populations.

Along the way, I have become the person who is open to working with Students of Color, and working with first-generation students, or working with families dealing with DACA or immigration issues. It's just been wonderful personally, and fulfilling professionally.

Claire, and several other veteran participants, had not only developed multicultural skills, but had become resources for matters related to race and ethnicity among their peers.

Participants demonstrated the multicultural competency development journey that evolves along a continuum of awareness, followed by knowledge and skills. Those student affairs educators who had been part of NCORE for several years reflected on their journey, where they had been and how they had developed. They recognized that even though they were veteran participants perceived as culturally competency campus role models, their multicultural competency journey was continuing.

\section{Discussion and Implications for Practice}

The research question for this study specifically addressed the second dimension of the MDCC that included multicultural awareness, knowledge, and skills. Findings indicated that the student affairs educators that participated in the NCORE experience developed multicultural competencies parallel to the second dimension of the MDCC including multicultural awareness, knowledge and skills. Participants developed awareness from the conference and knowledge to an extent. Participants gained some knowledge by attending NCORE, but as they reflected on their experience became aware they had more knowledge to gain. One weeklong conference did not provide the 
depth or breadth of information required for student affairs educators to consider themselves truly knowledgeable on issues related to race and ethnicity. Multicultural skills were not acquired until student affairs educators attended NCORE more than once and had the opportunity to apply the awareness and knowledge gained from the NCORE experience to their professional responsibilities.

Findings also indicated that multicultural competency development occurs along a continuum. Participants suggested that they first developed multicultural awareness, followed by knowledge and finally skills; simultaneously they focused on their personal development before their professional development. For example, only those participants who had attended more than one NCORE, returned to their professional positions and applied the multicultural awareness and knowledge that they had developed as part of NCORE to their work, were able to articulate multicultural skill development as part of their multicultural competencies related to the NCORE experience. Elena, who was attending her first NCORE suggested, “Skills, I don't have any answers. I think that's what the continual process is supposed to be."

\section{Implications for Practice}

If student affairs educators are to influence the campus environment and students in which they engage, they must have the multicultural competencies to do so. Participating in NCORE requires a significant investment in time and money, but findings from this study suggest that this investment is worthwhile. All participants from this study increased their multicultural competency because of attending NCORE.

Each year, NCORE participants include both first time participants and those who have participated for several years. First time attendees gained a significant amount of 
awareness and skills; multicultural competency continued to develop in participants who attended multiple times. This study reaffirms the value of NCORE for both types of participants. For institutions interested in developing the multicultural competency of their student affairs professionals, these findings support a strategy where institutions invite professionals who have not previously attended and also encourage professionals to attend multiple times. This approach helps institutions provide at least foundational awareness and knowledge to a larger percentage of their staff.

Participants who attended multiple years noted that it took several years engaging in the NCORE experience before they felt they had the competencies to influence others, suggesting the importance of encouraging at least a core group of staff members to attend multiple times. Therefore, those with a developed expertise can then serve as multicultural competent educators for other staff members. Additionally, because institutional change is more likely to occur when student affairs educators develop and apply their skills in their work, these staff may also take on more responsibility for creating socially just and inclusive environments.

We recognize that the multicultural competence of student affairs educators cannot be achieved solely through participation in one conference. However, NCORE, because of its focus, goals and conference structure, does provide a valuable vehicle by which multicultural competence can be developed. Many student affairs professionals may have had graduate coursework or have participated in other professional development sessions. Despite these prior experiences, all participants found NCORE to be a powerful and useful conference that enhanced their multicultural development. 


\section{Future Research}

Multicultural competency development and the role of NCORE in this development, is rich with opportunity for future research. First, a quantitative design might include more research participants from varied institutional types and geographical regions. Longitudinal studies investigating changes in multicultural awareness, knowledge, and skills of student affairs educators attending NCORE over time would further inform the continuum of multicultural competency development. Beyond student affairs educators, replicating this study for faculty or administrators might provide valuable findings regarding advancing institutional goals related to social justice, equity, diversity and inclusion. This study addressed the influence NCORE had on the second dimension of the MDCC related to awareness, knowledge and skills. Exploring the first and third dimensions of the MDCC such as varying identities and levels of development including personal, professional, organizational and societal would provide a deeper understanding of the multidimensional elements of multicultural competency in higher education. For example, this study did not investigate the difference and similarities by race and ethnicity. However, the lived experiences and understanding worldviews of others were salient elements of the NCORE experience and would be a valuable focus for future studies.

\section{Conclusion}

Institutions of higher education espouse goals of diversity, social inclusion and graduating students with the ability to navigate a global society (Bresciani, 2008). Student affairs educators play a significant role in meeting these institutional goals, but to be effective requires multicultural competence. Although student affairs educators 
may be exposed to these topics in graduate school (Cuyjet, Longell-Grice, \& Molina, 2009; Dickerson et al., 2011), acquiring multicultural competence is a developmental process. This study explored the experiences of student affairs educators developing multicultural competencies within the context of NCORE. Findings demonstrate that NCORE is a powerful experience that aids in the multicultural awareness, knowledge, and skills of student affairs professionals. 


\section{References}

Bresciani, M.L. (2008). Global competencies in student affairs/services professionals: A literature synthesis. College Student Journal, 42(3), 906-919.

Creswell, J.W. (2014). Research design: Qualitative, quantitative, and mixed methods approaches (4th ed.). Thousand Oaks, CA: Sage.

Cuyjet, M.J., Longell-Rice, R., \& Molina, E. (2009). Perceptions of new student affairs professionals and their supervisors regarding the application of competencies learned in preparation programs. Journal of College Student Development, 50(1), 104-119.

Davis, T., \& Harrison, L.M. (2013). Advancing social justice: Tools, pedagogies, and strategies to transform your campus. San Francisco, CA: Jossey-Bass.

Dickerson, A.M., Hoffman, J.L., Anan, B.P., Brown, K.F., Vong, L.K., Bresciani, M.J., Monzon, R., \& Oyler, J. (2011). A comparison of senior student affairs office and student affairs preparatory program faculty expectations of entry-level professional competencies. Journal of Student Affairs Research and Practice, 48(4), 463-479.

Harper, S.R. (2008). (Ed.). Creating inclusive campus environments: for cross-cultural learning and student engagement. National Association of Student Personnel Administrators.

Howard-Hamilton, M.F., Richardson, B.J., \& Shuford, B. (1998). Promoting multicultural education: A holistic approach. College Student Affairs Journal. 18 (1), 5-17.

Hurtado, S., \& Guillermo-Wann, C. (2013). Diverse Learning Environments: Assessment and creating conditions for student success - Final Report to the Ford Foundation. University of California, Los Angeles: Higher Education Research Institute.

Jones, S.R., Torres, V., \& Arminio, J. (2014). Negotiating the complexities of qualitative research in higher education: Fundamental elements and issues ( $2^{\text {nd }}$ ed.). New York, NY: Routledge.

King, P.M., \& Howard-Hamilton, M. (2003). An assessment of multicultural competence. NAPSA Journal, 40(2), 119-133.

Maxwell, J.A. (2013). Qualitative research design: An interactive approach (3rd ed.). Thousand Oaks, CA: Sage.

Merriam, S.B. (1997). Qualitative research and case study applications in education. Hoboken, NJ: John Wiley \& Sons.

NCORE. (2018). Request to attend the $31^{\text {st }}$ Annual National Conference on Race and Ethnicity in American Higher Education. Retrieved from: https://www.ncore.ou.edu/media/filer_public/3d/a4/3da4f276-0403-4b94-93d3bd62b7c9e37e/justification_letter_2018.pdf

Pope, R.L., \& Reynolds, A.L. (1997). Student affairs core competencies: Integrating multicultural awareness, knowledge, and skills. Journal of College Student Development. 38(3): 266-277.

Pope, R.L., Reynolds, A.L., \& Mueller, J.A. (2004). Multicultural competence in student affairs. San Francisco, CA: Jossey-Bass.

Pope, R.L., Reynolds, A.L., \& Mueller, J.A. (2014). Creating cultural change on campus. San Francisco, CA: Jossey-Bass.

Rossman, G.B. \& Rallis, S.F. (2012). Learning in the field: An introduction to qualitative research (3rd ed.). Thousand Oaks, CA: Sage. 
Journal Committed to Social Change on Race and Ethnicity | 2018

Saldaña, J. (2015). The coding manual for qualitative researchers. Thousand Oaks, CA: Sage.

Seidman, I. (2013). Interviewing as qualitative research: A guide for researchers in education and the social sciences. New York, NY: Teachers College Press.

Stake, R.E. (1995). The art of the case study design. Thousand Oaks, CA: SAGE Publications.

Sue, D. W. (2001). Multidimensional facets of cultural competence. The Counseling Psychologist, 29(6), 790-821.

Watt, S.K. \& Linley, J.L. (2013). Creating successful multicultural initiatives in higher education and student affairs. New Directions for Student Services. 144(1). San Francisco, CA: Jossey-Bass.

Yin, R.K., (2009). Case study research: design and methods. Thousand Oaks, CA: SAGE Publications. 